\title{
Exact parametric solutions for the first Painlevé nonlinear ODE
}

\author{
D. E. Panayotounakos, Th. I. Zarmpoutis* and G. Kostogiannis
}

School of Applied Mathematics and Physical Sciences, National Technical University of Athens, Zografou Street 5, Hellas, GR 15773.

*Corresponding author E-mail: zarmpoutis@tee.gr

\begin{abstract}
We present a mathematical methodology for constructing the exact parametric solution of the first Painlevé second order nonlinear ordinary differential equation. Several admissible functional transformations are introduced through an intermediary analysis delivering us from the a priori construction of power series solutions..
\end{abstract}

Keywords: Exact parametric solution, First Painlevé transcendent, Nonlinear ordinary differential equations.

\section{Introduction}

Painlevé equations are met in different fields of the contemporary physics. They resulted from solving the problem of extracting equation classes of the form $y_{x x}^{\prime \prime}=R\left(x, y, y_{x}^{\prime}\right)(R=$ any rational function) with analytic coefficient where integrals have no movable critical points. This problem was solved firstly by Painlevé [7]. For fifty extracted equations (listed [4]) in the six not integrated by the above researcher. These six equations are referred as Painlevé transcendents and their solutions are called as transcendental Painlevé functions. It was a controversial open problem for many years to prove that these six equations were really irreducible for generic values of the parameters. This was finally proved by Nishioha [6] and Umemura [13]. Extensive and thorough investigation for the Painlevé transcendents are included in [2].

The first of the abovementioned equations, tradionally called PE is a second order nonlinear ordinary differential equation (ODE) of the form $y_{x x}^{\prime \prime}=6 y^{2}+1$ (reducible case), well known also as $1^{\text {st }}$ Painlevé transcendent. The solution of this equation is unique and can be presented, in vicinity of movable pole $x_{0}$ in terms of the series [10]

$$
y(x)=\left(x-x_{0}\right)^{-2}+\frac{x}{10}\left(x-x_{0}\right)^{2}+\frac{1}{6}\left(x-x_{0}\right)^{3}+C\left(x-x_{0}\right)^{4}+\sum_{j=6}^{\infty} a_{j}\left(x-x_{0}\right)^{j} .
$$

Here $x_{0}$ and $C$ are arbitrary constants. The coefficients $\alpha_{j}(j \geq 6)$ are uniquely defined in terms of $x_{0}$ and $C$, and for large values of $|x|$ the asymptotic formula $y(x) \sim z^{1 / 2} P^{o}\left(4 x^{5 / 4} / 3-a ; 12, b\right)$ holds where $P^{o}$ represents the elliptic Weierstrass function.

In the present work we construct the exact parametric solutions of the first PE nonlinear ODE (redouble case), based on some of the results included in [8] and [9]. Introducing a series of Theorems, Propositions and Lemmas, several of which are new, we su cceed in constructing the exact parametric solutions (without any restrictive assumption) for the first PE. The main contributions of the current work is the introduction of mathematical methodology as well as the introduction and utilization of several convenient new admissible functional transformations, that allow the parametric solution of the PE through the contribution of solutions of an Emden-Folwer type ODE $y_{x x}^{\prime \prime}=F(x)\left(y_{x}^{\prime}\right)^{l}$; $F(x)=6 y^{2}+x ; y=f(x)$. 


\section{Some basic results}

In what follows the well known notation $y_{x}^{\prime}=d y / d x, y_{x x}^{\prime \prime}=d^{2} y / d x^{2}, \ldots$ is used for the total derivatives. For the sake of visualization, we use the symbolic notation $\{n, m, l\}$ to denote the generalized Emden-Fowler equation.

$$
y_{x x}^{\prime \prime}=A x^{n} y^{m}\left(y_{x}^{\prime}\right)^{l}
$$

Here we omit the insignificant parameter $A$, which can be reduced to \pm 1 by scaling the variables in accordance with the rule $x \rightarrow a x, y \rightarrow$ by, selecting the appropriate constants $a$ and $b$. Therefore, we have the Emden-Fowler equation

$$
y_{x x}^{\prime \prime}=x^{n} y^{m}\left(y_{x}^{\prime}\right)^{l}
$$

With these notations, the main results of this section can be phrased first with the

Theorem 2.1: For a given Emden-Fowler nonlinear ODE

$$
y_{x x}^{\prime \prime}=x^{n} y^{m}\left(y_{x}^{\prime}\right)^{l}
$$

the functional transformation

$$
z=\frac{x}{y} y_{x}^{\prime}, U=x^{n-l+2} y^{m+l-1}
$$

reduces (2.1) to the Abel nonlinear ODE of the second kind

$$
\left[z^{l} U-\left(z^{2}-z\right)\right] U_{z}^{\prime}=[(m+l-1) z+n-l+2] U .
$$

Furthermore, using the substitution

$$
\xi=U-z^{2-l}+z^{l-1},
$$

we obtain the Abel nonlinear ODE of the second kind

$$
\begin{gathered}
\xi \xi_{z}^{\prime}=[(m+2 l-3) z+n-2 l+3] z^{-1} \xi+ \\
+\left[(m+l-1) z^{2}+(n-m-2 l+3) z-n+l-2\right] z^{1-2 l}
\end{gathered}
$$

We postpone the proof of this theorem, because it is include in [11; p. 300-301] and we continue with the following

Proposition 2.2: If the variable coefficients of the Abel ODE

$$
\left[g_{1}(x) y+g_{0}(x)\right] y_{x}^{\prime}=f_{2}(x) y^{2}+f_{1}(x) y+f_{0}(x)
$$

satisfy the functional relation

$$
g_{0}\left(2 f_{2}+g_{1_{x}}^{\prime}\right)=g_{1}\left(f_{1}+g_{0_{x}}^{\prime}\right), \quad g_{1} \neq 0
$$

then its general solution is given by the formula 


$$
\frac{g_{1} y^{2}+2 g_{0} y}{g_{1} J}=2 \int \frac{f_{0}}{g_{1} J} d x+C,
$$

where $C$ is a integration constant and $J$ is the function

$$
J(x)=\exp \int \frac{2 f_{2}}{g_{1}} d x, \quad(J(x) \neq 0) .
$$

The Proposition 2.2 is extensively developed in [3,5].

Theorem 2.3: Consider the Emden-Fowler type second order nonlinear ODE

$$
y_{x x}^{\prime \prime}=f(x) y^{m}\left(y_{x}^{\prime}\right)^{l}
$$

where $m, l$ are arbitrary exponents and $f(x)$ is a smooth function of the independent variable, $-\infty<x<+\infty$. Then, the admissible functional transformation

$$
U=f^{3-l} y^{m+l-1}, \quad z=\frac{f}{y} y_{x}^{\prime} ; f=f(x), y \neq 0, m+l \neq 1
$$

where $z$ is a parameter such that $-\infty<z<+\infty$, transforms the nonlinear ODE (2.5) to the Abel type equation of the second kind

$$
U_{z}^{\prime}=\frac{U\left[(n-l+2) f_{x}^{\prime}+(m+l-1) z\right]}{U z^{l}+\left(f_{x}^{\prime}-z\right) z}, U z^{l}+\left(f_{x}^{\prime}-z\right) z \neq 0,
$$

Introducing also the convenient ad hoc assumption

$$
g(z)=f_{x}^{\prime}(x)
$$

where $g(z)$ is a smooth function to be determined, the above Abel equation becomes of the form

$$
\left(U z^{l}+[g(z)-z] z\right) U_{z}^{\prime}=U[(n-l+2) g(z)+(m+l-1) z]
$$

Applying the Julia construction given in Proposition (2.2), one evaluates the following parametric solution of the form

$$
\begin{gathered}
y^{3}=\frac{U_{z}^{\prime} z_{x}^{\prime}}{\left[(3-l) f_{x}^{\prime}+(m+l-1) z\right] f^{2-l}} ; \\
U_{z}^{\prime}=\frac{\left(g_{z}^{\prime}-1\right)-(l-1)(g-z)}{z^{l-1}}\left[-1 \pm \frac{\frac{g-z}{z^{l-1}}}{\sqrt{\left(\frac{g-z}{z^{l-1}}\right)^{2}+C}}\right] ; \\
g(z)=f_{x}^{\prime}(x)=z^{2(l-2)}\left[\frac{-m-2 l+3}{2(l-2)} z^{-2(l-2)}+C\right] ; \\
z_{x}^{\prime}=\frac{f_{x x}^{\prime \prime}}{g_{x}^{\prime}},
\end{gathered}
$$


where $C$ and $\stackrel{*}{C}$ are integration constants. The development and the proofs of this Theorem are developed in [8,9].

\section{Exact parametric solution of the First PE}

Consider the first nonlinear second order Painlevé ODE

$$
\begin{gathered}
y_{x x}^{\prime \prime}=6 y^{2}+x, \\
-\infty<x<+\infty, \infty<y<+\infty .
\end{gathered}
$$

Assuming that

$$
y=f(x), F(x)=-\left(6 y^{2}+x\right),
$$

where $f(x)$ is to be determined and introducing the admissible transformation

$$
y_{x}^{\prime}=\frac{1}{x_{y}^{\prime}} \Leftrightarrow y_{x x}^{\prime \prime}=-\frac{x_{y y}^{\prime \prime}}{x_{y}^{\prime 2}} ; x_{y}^{\prime} \neq 0
$$

the considered equation becomes to the following Emden-Fowler type nonlinear ODE with triad of notation $\{n, m, l\} \equiv\{1,0,3\}$, namely

$$
\begin{gathered}
x_{y y}^{\prime \prime}=F(x) x_{y}^{\prime 3} ; \\
F(x)=6 f^{2}(x)+x ; \\
y(x)=f(x) .
\end{gathered}
$$

Here $f(x)$ is the unknown smooth function to be determined.

We are able to introduce

Theorem 3.1: For a given nonlinear second order Emden-Folwer type ODE (with triad of exponents $\{n, m, l\} \equiv\{1,0,3\})$

$$
x_{y y}^{\prime \prime}=F(x) x_{y}^{\prime 3}
$$

there always exists an exact parametric solution $x=x\left(z, C_{1}, C_{2}\right) ; y=y\left(z, C_{1}, C_{2}\right)$, where $C_{1}$ and $C_{2}$ are suitable constants of integration and $z$ is the parameter $-\infty<z<+\infty$.

Proof. Combining (2.1) and (2.6) we state the admissible functional transformation

$$
\begin{gathered}
U=y^{2}, z=\frac{F(x)}{y} y_{x}^{\prime} ; \\
F(x)=6 f^{2}(x)+x=6 y^{2}(x)+x ; \\
y \neq 0, m+l \neq 1 ;-\infty<x<+\infty, \infty<y<+\infty
\end{gathered}
$$

where $f(x)$ is to be determined. Estimating the total differentials $d U$ and $d z$, the given nonlinear ODE

$$
x_{y y}^{\prime \prime}=F(x) x_{y}^{\prime 3},
$$


is reduced to the following nonlinear ODE (including $F_{x}^{\prime}$ ) $[8,9]$

$$
U_{z}^{\prime}=\frac{2 z U}{U z^{2}+\left(F_{x}^{\prime}-z\right) z}, \quad U z^{2}+\left(F_{x}^{\prime}-z\right) \neq 0
$$

The above leads to the result that the first order nonlinear ODE can be integrated, if the following convenient ad-hoc assumption

$$
g(z)=F_{x}^{\prime}(x)
$$

takes place. Here $g(z)$ is a new subsidiary function that have to be determined. Thus, ODE (3.2) becomes an Abel nonlinear ODE of the second kind namely

$$
\left[z^{3} U+[g(z)-z] z\right] U_{z}^{\prime}=2 z U
$$

or equivalently

$$
U_{z}^{\prime}=\frac{2 U}{z^{2} U+[g(z)-z]}, \quad z^{2} U+[g(z)-z] \neq 0 .
$$

This ODE is an Abel equation of the type (2.2) with $f_{1}=f_{2}=0$ and $f_{1}=1 ; g_{1}=z^{2} ; \quad g_{0}=g(z)-z$. Then, the functional relation (2.3) becomes

$$
z g_{z}^{\prime}-2 g+3 z=0
$$

performing the general solution

$$
g(z)=\stackrel{*}{C} z^{2}-\frac{3}{2} ; \stackrel{*}{C}=\text { first integration constant. }
$$

Thus, since in (3.4) $J=1 ; g_{1}=z^{3}$, the general solution of (3.4) is given by the functional relation (2.4)

$$
\frac{z^{3} U^{2}+2[g(z)-z] z U}{z^{3}}=C
$$

that is

$$
z^{2} U^{2}+2[g(z)-z] U-z^{2} C=0 ; \quad g(z)=C^{*} z^{2}-\frac{3}{2}
$$

Here, $C$ is a second integration constant. Therefore one finds $U(z)$ and $U_{z}^{\prime}(z)$ by the expressions

$$
\begin{gathered}
U(z)=-\frac{[g(z)-z]}{z^{l-1}} \pm \sqrt{\left\{\frac{[g(z)-z]}{z^{l-1}}\right\}^{2}+C} \\
g(z)=C z^{2}-\frac{3}{2}
\end{gathered}
$$

These formulae can be rewritten in the final form 


$$
\begin{gathered}
U(z)=\frac{-\left[2 C z^{2}-2 z-3\right] \pm \sqrt{\left(2 C z^{2}-2 z-3\right)^{2}+4 z^{4} C^{*}}}{2 z^{2}} ; \\
C, \stackrel{*}{C}=\text { suitable constants of integration. }
\end{gathered}
$$

Consequently, the first of equations (3.1) estimates directly the final result

$$
y(z)=f(x)=U^{2}=\left\{\frac{-\left[2{ }^{*} z^{2}-2 z-3\right] \pm \sqrt{\left(2 C^{*} z^{2}-2 z-3\right)^{2}+4 z^{4} C^{*}}}{2 z^{2}}\right\}^{2}
$$

while the independent variable $x(z)$ results as follows.

By the substitution $F(x)=6 y^{2}+x$ one gets $F_{x}^{\prime}=12 y y_{x}^{\prime}+1$, and from the second of (3.1) we have $y_{x}^{\prime} / y=z / F(x)=z /\left(6 y^{2}+x\right)$. Since $y=y(x)$ is determined through (3.1) as $y^{2}=U$, we extract

$$
\frac{y_{x}^{\prime}}{y}=\frac{z}{F(x)}=\frac{z}{6 y^{2}+x}=\frac{z}{6 U+x}
$$

by way of which we find

$$
y_{x}^{\prime}=\frac{z y}{6 U+x}= \pm \frac{z \sqrt{U}}{6 U+x}, 6 U+x \neq 0, U>0(\text { or } U<0) .
$$

On the other hand, by way of (3.3) as well as the expression for $F(x)\left(F=6 f^{2}+x\right)$ we estimate $F_{x}^{\prime}=-\left(12 y y_{x}^{\prime}+1\right)=\mp\left(12 \sqrt{U} y_{x}^{\prime}+1\right)=g(z)$, namely

$$
\left.y_{x}^{\prime}(x)= \pm \frac{g(z)-1}{12 \sqrt{U}}, x \neq 0, U>0 \text { (or } U<0\right), U \neq 0 .
$$

This functional relation affords the desired solution for $x=x(z)$, that is

$$
x(z)=\frac{12 z U(z)}{g(z)-1}-6 U(z)
$$

Both equations (3.6) and (3.7) furnish the demanded parametric solution. The possible elimination of the parameter $z$ among (3.6) and (3.7) results, furnishes an implicit functional relation that constitutes the desired solution in analytic form of the considered PE. We notice also that the prescribed elimination demands the investigation of the roots of quatric algebraic equations .

Finally, we establish the following

Lemma 3.2: Assume that $-\infty<x<+\infty ;-\infty<y<+\infty$ and $-\infty<x=$ parameter $<+\infty$. Then the original second order $1^{\text {st }} \mathrm{PE}$

$$
y_{x x}^{\prime \prime}=6 y^{2}+x,
$$




$$
\begin{aligned}
& y(z)=U^{2}(z) ; \\
& x(z)=\left[\frac{12}{g(z)-1}-6\right] U(z),
\end{aligned}
$$

where

$$
\begin{gathered}
g(z)=\stackrel{*}{C} z^{2}-\frac{3}{2} \\
U(z)=\frac{-\left[2^{*} C z^{2}-2 z-3\right] \pm \sqrt{\left(2{ }^{*} z^{2}-2 z-3\right)^{2}+4 z^{4} C^{*}}}{2 z^{2}} .
\end{gathered}
$$

In these equations $z \neq 0, C$ and $\stackrel{*}{C}$ are suitable constants of integration, while $U(z)>0$.

If $U(z)<0$, the square roots in absolute value are introduced by inserting the imaginary variable $i=\sqrt{-1}$.

Remark 1. From the above analysis, it is clear that the exact parametric solution of the first $1^{\text {st }} \mathrm{PE}$ it is not unique, but it can be divided to several solutions valid inside equal number of consecutive subintervals of the main interval. Then, since the solution must be smooth, matching of these solutions in each point of changing is obligatory. This can be expressed by the equality of the function's value and its derivative on the left and right sides on every subinterval.

Remark 2. In a similar way one can establish an exact parametric solution for the $2^{\text {nd }}$ Painlevé equation, which is the second order nonlinear ODE $y_{x x}^{\prime \prime}=2 y^{3}+x y+a$ ( $\alpha=$ suitable parameter). In this case one introduces $y=f(x)$ and $F(x)=2 y^{2}+x y+a=2 f^{3}(x)+x f(x)+a$. Then, the resulting new ODE becomes similar to the examined first Painelevé ODE.

\section{References}

[1] H. T. Davis,. Introduction to Nonlinear Differential and Integral Equations. Dover Publ. Inc., New York, 1962.

[2] A.S. Fokas, A.R. Its, A.A. Kaparev and V.Vu, Painlevé transcendents. The Riemman-Hilbert approach. Mathematical Surveys and Monographs, 128 Providence, R.I American Mathematical Society, 2006.

[3] G. Julia Exercices d'Analyse. Bd. 3, Paris, 1933.

[4] E.L. Ince, Ordinary Differential Equations. Dover Reprint, 1927.

[5] E. Kamke, Differentialgleichungen, Lösungsmethoden und Lösungen. Vol.1, B.G Teubeur, Stuttgart, 1977.

[6] N. Nishioha, A note on the transcendency of Painlevé's first transcendent. Nagoya Mathematical Journal . 109 (1988), pp. $63-67$

[7] P. Painlevé, Sur le équations differentials du second ordre a d' ordre superieur dont l'intégrale génerale est uniform. Acta Mathematica, 25 (1902), pp. 1-85.

[8] D. E. Panayotounakos, T. I. Zarmpoutis and C.I. Siettos, On the construction of the general closed-form solutions of standing waves of the cubic nonlinear Schrödinger equation. Arch. of Appl. Mech. DOI 10.1007/s00419-012-0658-9, Vol 82, 10 (2012), pp. 1557-1568.

[9] D.E. Panayotounakos and Th. Zarmpoutis, Construction of exact parametric or closed form solutions of some unsolvable classes of nonlinear ODEs, (Abel's nonlinear ODEs of the first kind and relative degenerate equations). Int J. of Math. and Math Sciences, DOI 10.1155/2011/ 387429, ID 387429 (2011), pp. 1-11.

[10] I. G. Petrovski and E.M. Landis, On the number of limit cycles of the equation $d u / d x=P(x, y) / Q(x, y)$ where $P$ and $Q$ are polynomials of the second degree, Math. Sbornik 10 (2) (1955), pp. 209-250.

[11] A.D. Polyanin and V.F. Zaitsev, Handbook of Exact Solutions for Ordinary Differential Equations, Chapman \&Hall/CRC Press, New York, 2003.

[12] H. Umemura, Painlevé equations and classical functions. Sugaku Expositions. 2 (2),(1998), pp. 231-252.

[13] H. Umemura, On the irreducibility of Painlevé differential equations. Sugaku Expositions. 11 (1),(1989), pp. 77-100. 